\title{
Analysis of Cluster based Target Tracking in Wireless Sensor Networks
}

\author{
Karthika Gopal \\ Post Graduate Scholar \\ Department of Computer Science and Engineering \\ Karunya University, India
}

\author{
Ramalakshmi Krishnamoorthy \\ Assistant Professor (SG) \\ Department of Computer Science and Engineering \\ Karunya University, India
}

\begin{abstract}
Wireless Sensor Networks (WSNs), is one of the most emerging technologies in recent years. Signal processing between sensor nodes to the base station is most important task for these networks. Target tracking in WSN mainly focuses on localization and object tracking. This paper addresses various cluster based target tracking techniques in wireless sensor networks. To support reliability, energy efficiency and efficient routing, nodes are often grouped into clusters. The clusters can be formed before the intrusion enters the network which is called the static method or clusters are created by using signal strength from target which is called the dynamic method. This paper gives a various cluster based target tracking methods for efficient target tracking in WSN.
\end{abstract}

\section{Keywords}

Clustering, Static clustering, Dynamic clustering, Target tracking, Hybrid target tracking, Tree based target tracking.

\section{INTRODUCTION}

A wireless sensor network is a set of sensing agents deployed in a given area that form a network with wireless links and are capable of detecting, measuring, collecting, and processing their observations. WSNs consist of thousands of tiny sensor nodes deployed in a physical environment. Sensor nodes have been deployed to play an important roles in the applications of area monitoring, environmental/earth monitoring, industrial monitoring, agriculture, structural monitoring, passive localization and tracking in recent years. The above applications require target tracking for processes and events of interest occurring in an environment. A target tracking in WSNs can have several advantages (i) qualitative and fidelity observations (ii) signal processing accurately and timely and (iii) increased system robustness and tracking accuracy. The sensed data is composed, processed and then routed to the desired end user through a designated base station [11]. To improve the quality of tracking, sensors need to make accurate estimates of the location of targets. The important characteristics of the WSN are that power consumption constrains for nodes using batteries or energy harvesting, ability to cope with node failures, mobility of nodes, heterogeneity of nodes, scalability to large scale of deployment, ability to withstand harsh environmental conditions, ease of use and power consumption. Target tracking applications can be cluster based, tree based, and hybrid based techniques. In a target tracking application, the sensor nodes which can sense the target at a specified time are kept in active mode while the remaining nodes are to be retained in sleep mode so as to consume energy until the target reaches them.
Target tracking in course of maintaining the balance between network resources like energy and bandwidth. In tree based target tracking the nodes that detect the target communicates with each other and selects a root node. The root node collects information from all the nodes via a spanning tree. If the root node is far away from the target, then the tree will be reshaped. There will be more accurate object tracking in spanning tree based approaches, tree organizations result in high-energy consumptions. Hybrid methods are the tracking algorithms that accomplish the requirements of more than one type of object tracking. In cluster based networks, nodes are either classified as cluster members or cluster heads. In cluster based target tracking, member nodes detect the target and send the information to their cluster head. Cluster heads collect all information from their clustered nodes and estimate the position of the target by using localization techniques. After the position of the target is calculated, cluster head sends the position information to the sink. One of the important benefits is the reducing the energy consumption in the cluster based approaches.

Cluster based target tracking algorithms can be further divided into two groups: static and dynamic approaches. In Static Clustering, clusters are formed statically at the time of network deployment.

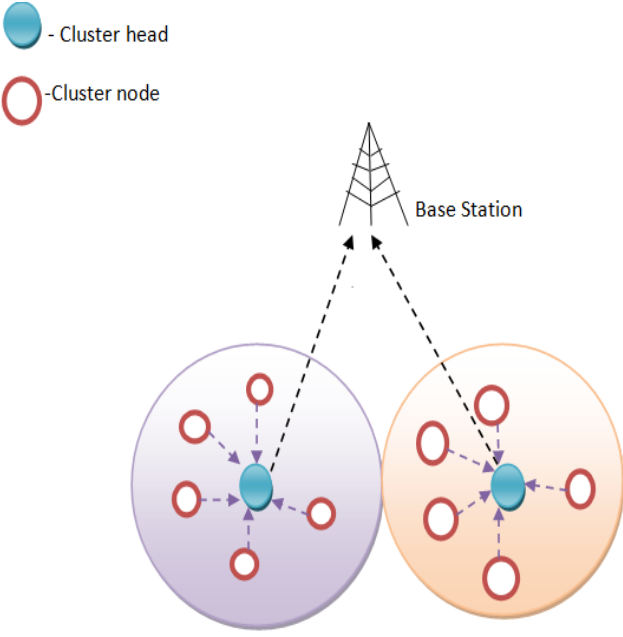

Fig.1: Clustering of sensors in network.

The attributes of each cluster, such as the size of a cluster, the area it covers, and the nodes it possesses, are static. The clusters are dynamically constructed in dynamic clustering model. The clusters are formed in dynamic approach, while the nodes sense the target in the network. The paper has been classified as follows. Section II discuss about concept of basic 
clustering. Section III discuss about classification of target tracking techniques. Section IV discuss about techniques in cluster based target tracking.

\section{CLUSTERING}

In order to support processing of data through efficient network, sensor nodes can be divided into a number of small groups called clusters. The grouping of sensor nodes into clusters is called clustering. Every cluster would have a head, commonly referred to as cluster-head $(\mathrm{CH})$. A $\mathrm{CH}$ may be elected by the sensor node with maximum residual energy. There are several advantages in clustering phenomenon. The basic advantage is that, it supports network scalability.

It can localize the route setup within the cluster. Clustering can also preserve communication bandwidth. Moreover, clustering can make stable the network topology at the level of sensors. The $\mathrm{CH}$ can also implement more effective management strategies to elongate the battery life of the individual sensors and to maximize the network lifetime. The activated sensors broadcast their residual energy level in the cluster.

The sensor with the maximum residual energy is elected as the cluster head $(\mathrm{CH})$ to take charge of signal processing.

Fig.1 shows the basic clustering concept. The clustered detecting sensors transfer their observations to the $\mathrm{CH}$. The cluster head further process the information to the sink, referred to as the base station.

\section{Benefits of clustering}

- Provides useful energy consumption

- Provides scalability for large number of nodes

- Reduces communication overhead for both single and multi hop.

\section{CLASSIFICATION OF TARGET TRACKING METHODS}

Target tracking in WSN can be mainly classified into two categories[3]. Hierarichal and peer to peer networks. The hierarichal network is a mesh based system having multihop connectivity among nodes that are deployed. The sensors that monitor the event and report back to the base station. The hierarichal network is classified into three main categories Fig.2. Tree based, Cluster based and Hybrid based methods. In this paper we discuss about the cluster based tracking methods. The cluster based tracking method can be further divided into static and dynamic clustering schemes.

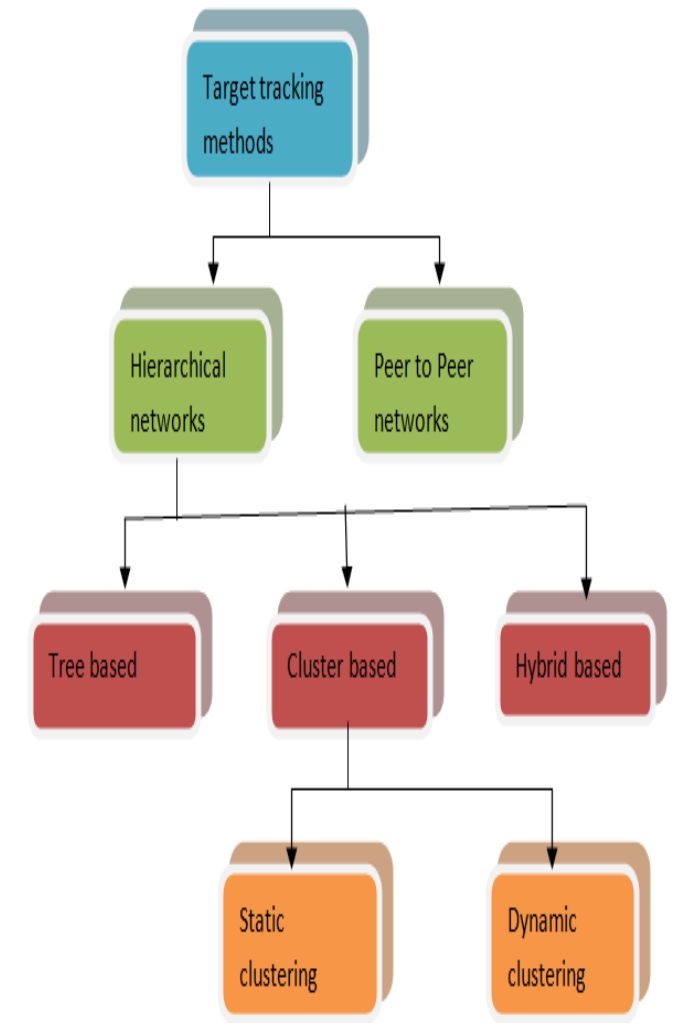

Fig 2: Classification Target Tracking Methods

\section{TWO TYPES OF CLUSTER BASED TARGET TRACKING METHODS}

\subsection{Static Cluster Based Target Tracking}

In static cluster based target tracking the clusters are formed static at the time of network deployment. The clustering nodes and cluster head are defined static before target tracking. Fig.3 illustrates that once the target enters the WSN the clusters that are activated, only the sensors which sense the presence of the target. And the nodes that sense the target transfer their observations to the cluster head $(\mathrm{CH})$.

The clusterhead that estimate the location of the target and predict the next location of the target. By using location information it predicts the future position of the target in a given period of time. The current cluster head sends information to the nearest cluster head, that the predicted next location of the target be ready to detect the target.

However the static clustering have some drawbacks inspite of its simplicity. It is not robust because of its fixed clusters and clusterhead. If the cluster head dies due to power depletion then the cluster provide useless. Because of static cluster the sensor nodes from different clusters cannot share information and collaborate on data processing. 


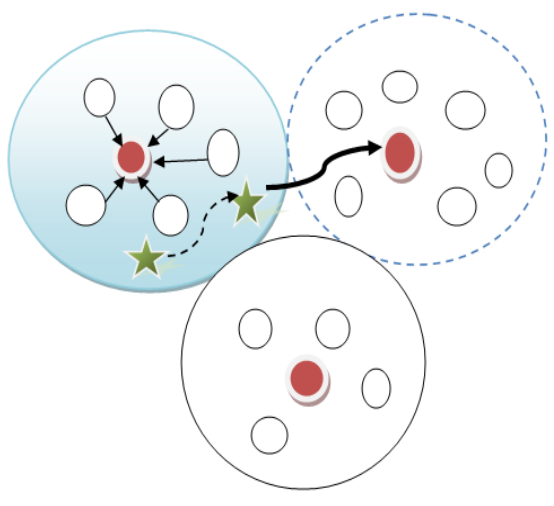

Fig 3: Static cluster based target tracking.

\subsection{Dynamic Cluster Based Target Tracking}

Dynamic clustering sensors that forms different clusters at different times according to the movement of the target. So that the node can be a member of different clusters at different time and it minimizes the localization error[1]. Unless static clustering, dynamically it forms the cluster with sensors. Only one cluster is active at each time in the accordance of target moving manner. Thus it reduces the energy consumption of sensors in the cluster. It is more reliable than static clustering method. Also the sensor nodes sensing and forwarding redundant information has been reduced.

Fig.4 illustrates when the intrusion enters the WSN, only the sensors that detect the presence of the target forms a cluster dynamically[2]. The node with maximum residual energy elected as a cluster head and the sensed information by the cluster nodes transfer to the cluster head $(\mathrm{CH})$. The $\mathrm{CH}$ that calculates the location and trajectory of the target based on the observations and predict the next location of the target. And it sends the information to the node closest to the targets predicted future location. This node acts as a $\mathrm{CH}$ and forms the cluster with its neighbouring nodes.

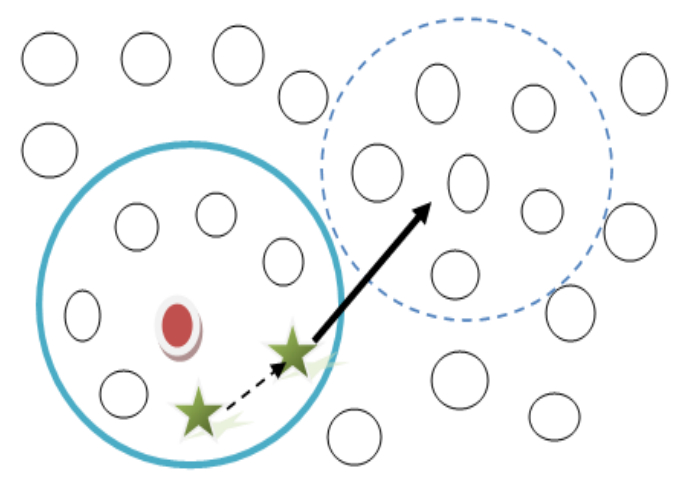

Fig 4: Dynamic cluster based target tracking.

The dynamic clustering supports to form different clusters at different times.Its communication range is significantly higher than their sensing range and also it reduces the amount of redundant data in the network.
Table 4.1 Comparison table of static and dynamic clustering.

\begin{tabular}{|l|l|l|}
\hline Parameters & Static & Dynamic \\
\hline $\begin{array}{l}\text { Localization } \\
\text { error }\end{array}$ & Maximum & Minimum \\
\hline $\begin{array}{l}\text { Cluster head } \\
\text { selection }\end{array}$ & $\begin{array}{l}\text { Statically } \\
\text { defined }\end{array}$ & $\begin{array}{l}\text { Maximum } \\
\text { residual energy }\end{array}$ \\
\hline $\begin{array}{l}\text { Cluster } \\
\text { formation }\end{array}$ & Simple & Complex \\
\hline $\begin{array}{l}\text { Energy } \\
\text { consumption }\end{array}$ & High & Low \\
\hline Reliability & Reliable & More reliable \\
\hline Redundant data & More & Less \\
\hline Robustness & Not robust & Robust \\
\hline
\end{tabular}

\section{CONCLUSION}

Thus the analysis of cluster based target tracking with the classification of static and dynamic cluster based target tracking methods are discussed. The clusters are formed to support reliability, energy efficiency of the nodes in the network. Dynamic clustering provides more reliability and energy efficiency than the static clustering method, because in static clustering clusters constructed and nodes it possess are static.

\section{REFERENCES}

[1] W. Yang, Z. Fu, J. Kim, and M.-S. Park," An Adaptive Dynamic Cluster-Based Protocol for Target Tracking in Wireless Sensor Networks", in Proc. of. WAIM 07, 2007, pp. 157-167.

[2] W. Chen, and J. Hou, "Dynamic Clustering for Acoustic Target Tracking in Wireless Sensor Networks", IEEE Transactions on Mobile Computing, 2004, pp. 258-271.

[3] Mohsin Fayyaz, Wireless Sensor Network, 2011, "Classification of Object Tracking Techniques in Wireless Sensor Networks".

[4] Feng Zhao1 and Jaewon ShinEt al IEEE Signal Processing Magazine, March 2002, "Information- Driven Dynamic Sensor Collaboration for Tracking Applications “.

[5] M. Walchli, P. Skoczylas, M. Meer and T. Braun, "Distributed Event Localization and Tracking with Wireless Sensors," in Proceedings of the 5th international Conference on Wired/Wireless internet Communications, May 23 - 25, 2007.

[6] E. Olule, G. Wang, M. Guo and M. Dong, "RARE: An Energy Efficient Target Tracking Protocol for Wireless Sensor Networks," 2007 International Conference on Parallel Processing Workshops (ICPPW 2007).

[7] Shashi Phoha Et al International Journal of Distributed Sensor Networks, 1: 81-99, 2005”Space-time Coordinated Distributed Sensing Algorithms for Resource Efficient Narrowband Target Localization and Tracking".

[8] R. R. Brooks, P. Ramanathan, and A. M. Sayeed, "Distributed Target Classification and Tracking in Sensor Networks", IEEE Signal Processing Magazine , vol. 91, 2002, pp. 1163-1171. 
[9] H. Yang, and B. Sikdar, "A Protocol for Tracking Mobile Targets Using Sensor Networks", in Proc. of SNPA 03, 2003, pp. 7181.

[10] Elham Ahmadi, Masoud Sabaei, Mohamad Husain Ahmadi "A New Adaptive Method for Target Tracking in Wireless Sensor Networks", in International Journal of
Computer Applications (0975 - 8887), Volume 22No.9, May 2011.

[11] V. Mhatre and C. Rosenberg. Design guidelines for wireless sensor networks: communication, clustering and aggregation. Ad Hoc Networks, 2(1):45-63,2004. 